\title{
Documentos
}

\section{Pautas de manejo del embarazo múltiple complicado con la muerte fetal in útero de un gemelo}

\author{
Susana Barba J. ${ }^{1}$, Jorge Carvajal C. ${ }^{2} \mathrm{PhD}$. \\ 1 Programa de Posgrado de Obstetricia y Ginecología, Facultad de Medicina, Pontificia Universidad Católica de Chile, \\ 2 Unidad de Medicina Materno Fetal, División de Obstetricia y Ginecología, Facultad de Medicina, Pontificia Universidad \\ Católica de Chile.
}

\section{RESUMEN}

El embarazo múltiple está asociado a un aumento en la mortalidad y morbilidad perinatal, comparado con el embarazo único. Una complicación grave que afecta al embarazo gemelar es la muerte fetal in útero de un gemelo, situación que implica un riesgo de morbilidad/mortalidad para el feto sobreviviente y la embarazada. En base a la mejor evidencia disponible sugerimos una pauta de manejo que permita optimizar el resultado perinatal del gemelo sobreviviente y de la embarazada.

\section{PALABRAS CLAVE: Embarazo gemelar, muerte de un gemelo, feto sobreviviente}

\section{SUMMARY}

Twin pregnancy is associated with increased perinatal mortality and morbidity compared with singleton pregnancy. A serious complication in a twin pregnancy is the intrauterine fetal demise of one fetus; this situation involves a larger risk of morbidity and mortality for the surviving fetus and the pregnant woman. Based in the best available evidence we suggest a management guideline to optimize the outcomes of the surviving twin and the pregnant women.

\section{KEY WORDS: Twin pregnancy, single fetal demise, surviving fetus}

\section{INTRODUCCIÓN}

El embarazo múltiple constituye en nuestros días una porción creciente de embarazos a nivel mundial, debido a la expansión de los tratamientos de infertilidad y a la edad tardía de inicio de la maternidad $(1,2)$. En el Hospital Clínico de la Universidad de Chile, la tasa de embarazo gemelar fue $1,96 \%$ para el periodo $1998-2000$ y $2,63 \%$ para el periodo 2001-2004, lo que representa un aumento del 30\% (3). Datos no publicados del Hospital Clínico de la Pontificia Universidad Católica de Chile, muestra un aumento en la tasa de embarazos gemelares de $0,86 \%$ en el año $2008,1,16 \%$ en 2009 y $1,21 \%$ en 2010 .

El embarazo múltiple es un embarazo de alta complejidad, asociado a una mayor morbilidad y mortalidad perinatal, comparado con embarazos únicos. Una de sus complicaciones es la muerte de un gemelo in útero, descrito hasta en el $6,2 \%$ de los 
gemelos. El riesgo de muerte in útero de un gemelo es hasta tres veces mayor en el embarazo monocorial comparado con el bicorial (4-6).

El evento de la muerte de un gemelo in útero es un problema clínico grave, pero poco frecuente, explicando la escasa información que orienta el manejo, principalmente en series de casos. El objetivo de este artículo, a propósito de un caso clínico, es crear una pauta de manejo uniforme en embarazos múltiples complicados por la muerte de un gemelo, en base a la mejor evidencia disponible.

Caso clínico. Primigesta de 28 años, cursando un embarazo gemelar bicorial biamniótico con diagnóstico ecográfico a las 9 semanas de edad gestacional (EG). Control prenatal adecuado y de inicio temprano, que se complica a las 16 semanas con una restricción de crecimiento fetal selectiva (RCFs) y posteriormente con la muerte del segundo gemelo a las 26 semanas de embarazo, momento en que se decide manejo expectante, con control ecográfico fetal seriado para evaluar bienestar fetal en el gemelo sobreviviente y control de analítica sanguínea materna por riesgo de coagulación intravascular diseminada (CID) (Tablas I, II y III). Dado el bienestar fetal del gemelo sobreviviente y de la embarazada, el embarazo se prolonga hasta las 37 semanas momento en que se efectúa inducción de parto. Se obtuvo parto vaginal, con recién nacido femenino, con examen físico general normal, peso 2.815 gramos, talla 45 centímetros, Apgar 9-10. El seguimiento neurológico del recién nacido al mes del nacimiento es calificado como normal. Al momento del parto se constató la presencia de feto papiráceo de 65 gramos (Figura 1).

\section{MATERIAL Y MÉTODO}

Se realizó la búsqueda en TripDataBase, Cochrane, MEDLINE y Scholar Google, usando la combinación de palabras: "single twin demise", "single twin death", "fetal demise in a twin pregnancy", "single survivors", "single fetal demise", "single intrauterinedeath", "intrauterine death of a twin", "single fetal death" y "surviving co-twin"; además, se usó la función Mesh: ("Pregnancy, Multiple"[Mesh]) AND "Fetal Death"[Mesh] y ("Fetal Death"[Mesh]) AND "Twins"[Mesh]. Se incluyó los estudios con texto en idioma inglés y español.

Se encontraron 46 estudios observacionales, obteniéndose información completa de 25 (2 en español); además se obtuvo una revisión sistemática y dos narrativas, estos artículos constituyen las referencias primarias. Se revisó los capítulos de embarazo múltiple en: UpToDate online y Obstetricia de Williams, y algunos trabajos citados en la bibliografía de artículos incluidos, como referencias secundarias.

\section{DEFINICIONES}

Gemelo evanescente. Corresponde a la reabsorción del gemelo muerto, generalmente se produce cuando se realiza un diagnóstico ecográfico temprano de un embarazo múltiple, usualmente antes de las 14 semanas, y posteriormente en las siguientes evaluaciones ecográficas se encuentra un único embrión o feto (7-9).

Tabla I

\section{SEGUIMIENTO ECOGRÁFICO DEL EMBARAZO GEMELAR PREVIO A LA} MUERTE FETAL DE UN GEMELO

\begin{tabular}{|c|c|c|c|c|c|c|c|c|c|c|c|c|c|c|}
\hline$\overline{\text { Edad }}$ & $\begin{array}{l}\text { sem } \\
\text { sl }\end{array}$ & anas & $\begin{array}{r}12 \\
\text { seme } \\
\mathrm{Gl}\end{array}$ & $\begin{array}{l}+5 \\
\text { anas } \\
\text { Gll }\end{array}$ & & $\begin{array}{c}6+5 \\
\text { רanas } \\
\text { Gll }\end{array}$ & $\begin{array}{r}19 \\
\text { sem } \\
\text { Gl }\end{array}$ & $\begin{array}{l}+2 \\
\text { anas } \\
\text { Gll }\end{array}$ & $\begin{array}{l}21 \\
\text { sem } \\
\text { Gl }\end{array}$ & $\begin{array}{l}+5 \\
\text { anas } \\
\text { Gll }\end{array}$ & $\begin{array}{c}24 \\
\text { sem } \\
\text { Gl }\end{array}$ & $\begin{array}{l}+2 \\
\text { anas } \\
\text { Gll }\end{array}$ & $\begin{array}{c}\text { sem } \\
\mathrm{Gl}\end{array}$ & Gil \\
\hline Latido & $(+)$ & $(+)$ & $(+)$ & $(+)$ & $(+)$ & $(+)$ & $(+)$ & $(+)$ & $(+)$ & $(+)$ & $(+)$ & $(+)$ & $(+)$ & $(-)$ \\
\hline $\begin{array}{l}\text { Biom } \\
\text { (seme }\end{array}$ & 9 & 9 & $12+5$ & $12+5$ & $16+5$ & $14+5$ & $19+2$ & $16+2$ & $21+5$ & 18 & $24+2$ & 18 & 26 & - \\
\hline Líquido amniótico & $\mathrm{N}$ & $\mathrm{N}$ & $\mathrm{N}$ & $\mathrm{N}$ & $\mathrm{N}$ & $\downarrow$ & $\mathrm{N}$ & $\downarrow$ & $\mathrm{N}$ & $\downarrow$ & $\mathrm{N}$ & $\mathrm{OHA}$ & $\mathrm{N}$ & - \\
\hline Arteria umbilical & $\mathrm{NE}$ & $\mathrm{NE}$ & NE & NE & $\mathrm{N}$ & FAD & $\mathrm{N}$ & FAD & $\mathrm{N}$ & FAD & $N$ & FAD & $\mathrm{N}$ & - \\
\hline Anatomía & $\mathrm{NE}$ & $\mathrm{NE}$ & TN 1.2 & TN 1.5 & $\mathrm{~N}$ & $\mathrm{~N}$ & $\mathrm{~N}$ & $\mathrm{~N}$ & $\mathrm{~N}$ & $\mathrm{~N}$ & $\mathrm{~N}$ & $\mathrm{~N}$ & $\mathrm{~N}$ & \\
\hline
\end{tabular}

GI: Gemelo I. GII: Gemelo II. (+): Presente. (-): Ausente. N: Normal. $\downarrow$ Disminuido. FAD: Flujo ausente en diástole.

TN: translucencia nucal. NE: No evaluado. 
Tabla II

SEGUIMIENTO ECOGRÁFICO DEL EMBARAZO GEMELAR DEL FETO SOBREVIVIENTE

\begin{tabular}{lccccccc}
\hline $\begin{array}{l}\text { Edad } \\
\text { gestacional }\end{array}$ & $\begin{array}{c}28 \\
\text { semanas }\end{array}$ & $\begin{array}{c}29 \\
\text { semanas }\end{array}$ & $\begin{array}{c}29+5 \\
\text { semanas }\end{array}$ & $\begin{array}{c}32+5 \\
\text { semanas }\end{array}$ & $\begin{array}{c}33+5 \\
\text { semanas }\end{array}$ & $\begin{array}{c}34+5 \\
\text { semanas }\end{array}$ & $\begin{array}{c}35+5 \\
\text { semanas }\end{array}$ \\
\hline EPF (gramos) & 1200 & - & 1390 & - & 2274 & - & 2981 \\
Percentil & $75-90$ & - & $50-75$ & - & $50-75$ & - & $>90$ \\
Líquido amniótico & $\mathrm{N}$ & $\mathrm{N}$ & $\mathrm{N}$ & $\mathrm{N}$ & $\mathrm{N}$ & $\mathrm{N}$ & $\mathrm{N}$ \\
Arteria umbilical & $\mathrm{N}$ & $\mathrm{N}$ & $\mathrm{N}$ & $\mathrm{N}$ & $\mathrm{N}$ & $\mathrm{N}$ & $\mathrm{N}$ \\
ACM & $\mathrm{N}$ & $\mathrm{N}$ & $\mathrm{N}$ & $\mathrm{N}$ & $\mathrm{N}$ & $\mathrm{N}$ & $\mathrm{N}$ \\
\hline
\end{tabular}

N: Normal. ACM: Arteria cerebral media.

Tabla III

EXÁMENES DE SANGRE DEL SEGUIMIENTO MATERNO DESPUÉS DE LA MUERTE FETAL DE UN GEMELO

\begin{tabular}{lcccccc}
\hline Normal en embarazo & $\begin{array}{c}\text { Dímero D } \\
<\text { i00ng/mL }\end{array}$ & $\begin{array}{c}\text { TP } \\
70-120 \%\end{array}$ & INR & $\begin{array}{c}\text { TTPK } \\
26-40 \text { seg }\end{array}$ & $\begin{array}{c}\text { Fibrinógeno } \\
260-600 \\
\mathrm{mg} / \mathrm{dL}\end{array}$ & $\begin{array}{c}\text { Plaquetas } \\
140-400 / 103 \\
\mathrm{~mm}\end{array}$ \\
\hline $03-03-2011$ & 983 & 100 & 1,0 & 26,2 & 542 & 273 \\
$09-03-2011$ & 901 & 112 & 0,9 & 26,2 & 556 & 282 \\
$16-03-2011$ & 1010 & 103 & 1,0 & 27,6 & 696 & 296 \\
$23-03-2011$ & 1126 & 109 & 1,0 & 28,7 & 580 & 332 \\
$30-03-2011$ & 973 & 96 & 1,0 & 27,1 & 326 & 269 \\
$06-04-2011$ & 930 & 115 & 0,9 & 25,0 & 520 & 253 \\
$13-04-2011$ & 1231 & 102 & 1,0 & 28,6 & 561 & 233 \\
$20-04-2011$ & 1201 & 107 & 1,0 & 24,1 & 544 & 284 \\
$27-04-2011$ & 1352 & 112 & 1,0 & 23,3 & 533 & 246 \\
$04-05-2011$ & 1277 & 96 & 1,0 & 24,3 & 544 & 229 \\
$11-05-2011$ & 1426 & 112 & 1,0 & 23,6 & 463 & 22 \\
\hline
\end{tabular}

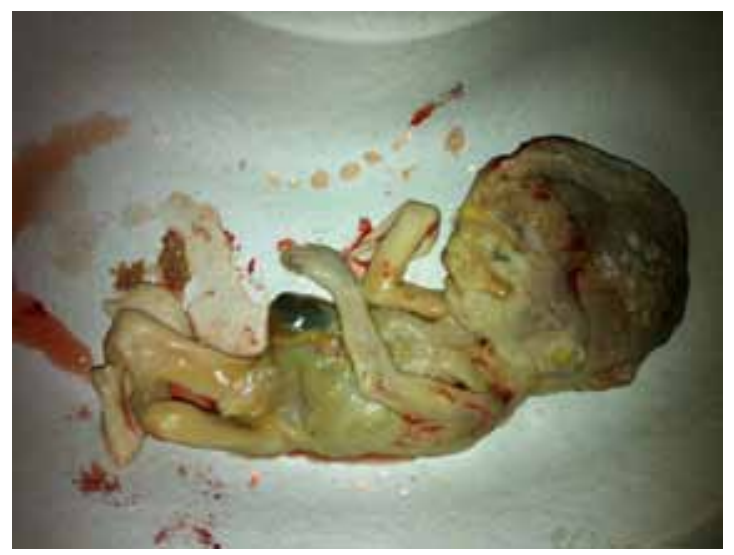

Figura 1. Feto papiráceo.
Feto papiráceo. Ocurre con la muerte de un gemelo a partir del segundo trimestre, con reducción a un gemelo momificado por compresión, que puede pasar inadvertido dentro de la membrana corioamniótica. Está asociado a un tiempo prolongado (pero no estimado) entre la muerte de un gemelo y el parto del sobreviviente (9-11).

Maceración. Necrosis estéril de un feto muerto, que lleva a la disolución parcial del mismo dentro de la cavidad amniótica (12). Se constata en caso de feto muerto in útero del tercer trimestre.

\section{EPIDEMIOLOGÍA}

La incidencia de muerte de un gemelo in útero se 
reportó desde 2,32\% hasta 6,2\% (8-10,13-15), valores que difieren según la corionicidad. Así, el embarazo monocorial tiene mayor riesgo de mortalidad comparado con el embarazo bicorial, con RR 10,9 para la muerte de los dos fetos; 3,8 para la muerte de un feto y un neonato (mortineonato), 1,3 para la muerte de un feto con un neonato vivo y 2,3 para dos neonatos muertos (16). Además, existe una diferencia en el riesgo de mortalidad de un gemelo por edad gestacional. El riesgo de muerte de un gemelo en el primer trimestre es $29 \%(7,17)$, a diferencia del riesgo estimado después de las 20 semanas descrito entre $3,7-5 \%(18,19)$.

\section{ETIOLOGÍA}

Las causas de muerte intrauterina de un gemelo son múltiples; éstas pueden ser sistematizadas en causas fetales, ovulares y maternas. Se estima que la causa es desconocida hasta en el $25 \%$ de los casos (13).

Fetal. Las causas fetales más frecuentemente reportadas como origen de la muerte de un gemelo in útero son el síndrome de transfusión feto fetal (STFF), las malformaciones o síndromes fetales, las infecciones y la restricción de crecimiento fetal (RCF)(14,20,21). Lamentablemente, existen limitantes para aclarar la causa de muerte fetal, habitualmente solo la mitad de los fetos son enviados a estudio de anatomía patología y el $62,5 \%$ de ellos estaban macerados (14).

Ovular. Estas causas se presentan con menor frecuencia e incluyen: infarto placentario, corioamnionitis, hematoma retroplacentario por desprendimiento prematuro de placenta normoinserta (DPPNI), inserción velamentosa del cordón y nudo verdadero de cordón $(10,14,15,22)$.

Materna. Las patologías maternas que pueden llevar a la muerte fetal, incluyen los síndromes hipertensivos del embarazo, diabetes gestacional y trombofilias hereditarias o adquiridas $(14,23)$.

\section{FISIOPATOLOGÍA DEL DAÑO AL GEMELO SO- BREVIVIENTE}

La morbilidad y mortalidad fetal del gemelo sobreviviente ha sido explicada por dos teorías, la tromboembólica y la hemodinámica.

1. Teoría tromboembólica. Esta teoría explica la liberación de elementos tromboembólicos desde el gemelo muerto hacia el gemelo sobreviviente, por medio de las anastomosis vasculares placentarias, que ocluyen vasos sanguíneos arteriales y causan daño por isquemia en los tejidos fetales, principal- mente riñones y corteza cerebral (24), además de aumentar el riesgo de una CID (23).

2. Teoría hemodinámica. Propone que la pérdida de resistencia vascular en el feto enfermo, lleva a una exanguinación del feto sobreviviente desde el momento pre mortem hasta un tiempo pos mortem no bien definido. Esta situación causa una anemia severa, con hipoperfusión tisular y daño de múltiples órganos fetales, en el gemelo sobreviviente (5,25-27). Un estudio de 8 embarazos gemelares complicados con la muerte de un gemelo (28), midió el hematocrito en cinco gemelos enfermos previo a la muerte y en cuatro de sus compañeros, y en los tres embarazos restantes se evaluó solo en el gemelo sobreviviente dentro de las primeras 24 horas. El promedio de hematocrito previo a la muerte fetal fue de $36 \%$ en los gemelos enfermos y $37 \%$ en los gemelos sanos; mientras en el grupo de gemelos sobrevivientes el hematocrito promedio fue $21 \%$. En el único caso en que se logró obtener una muestra del feto sobreviviente antes y después de la muerte de su compañero, se confirmó que existe una anemia aguda, donde el hematocrito disminuyó en $23 \%$ dentro de las primeras 24 horas pos mortem (28), confirmando que en algún momento, no bien establecido actualmente, se produce una anemia severa que lleva al daño tisular del feto vivo.

\section{CONSECUENCIAS FETALES}

La muerte de un gemelo in útero ocasiona consecuencias en el feto sobreviviente, y con menor frecuencia en la embarazada.

Consecuencias en el feto sobreviviente. La muerte de un gemelo in útero provoca desórdenes multiorgánicos en el gemelo sobreviviente, con una mortalidad descrita para el segundo feto desde 12,5-27\% $(5,29,30)$. Sin embargo, es importante mencionar que no toda alteración en el recién nacido es consecuencia de la muerte intrauterina del hermano, por ejemplo, un artículo reportó que el $48,4 \%$ de los recién nacidos presentan secuelas derivadas de la prematurez (14).

En particular, las lesiones neurológicas afectan con mayor frecuencia al feto sobreviviente (72\%). Las alteraciones del sistema nervioso central descritas son: quistes porencefálicos, encefalopatía multiquística, hidrocefalia, microcefalia, hidranencefalia, necrosis del cerebelo y necrosis medular, responsables de los mayores problemas psicomotores en el gemelo sobreviviente (31).

Un análisis de datos retrospectivos de 97 casos, reportó que la prevalencia de parálisis cerebral en el gemelo sobreviviente fue 93/1000 nacidos vivos (32), datos similares se encontraron en un análisis 
de 246 recién nacidos, donde la prevalencia fue $83 / 1000$ nacidos vivos (33). Como punto de comparación, debemos mencionar que en embarazos gemelares no complicados con la muerte de un gemelo y en embarazos únicos, la prevalencia de parálisis cerebral es 11 y 1 por 1000 nacidos vivos, respectivamente (34).

Otras alteraciones descritas en el feto sobreviviente con menor frecuencia tras la muerte de un gemelo in útero, son alteraciones de la frecuencia cardiaca, sufrimiento fetal, RCF, infección, eritroblastosis fetal, hemorragia placentaria, necrosis cortical renal, infarto esplénico y lesiones de piel (35).

El riesgo para el gemelo sobreviviente de presentar las consecuencias descritas, puede ajustarse según la edad gestacional en que ocurre la muerte fetal y la corionicidad del embarazo.

1. Edad gestacional: En un estudio retrospectivo, 43 embarazos gemelares complicados con una muerte fetal fueron evaluados según el trimestre en que ocurrió la muerte del feto (29). El primer grupo con EG menor de 16 semanas, el embarazo continuó sin complicaciones (11 casos), sin embargo, los grupos en que la muerte ocurrió en el segundo y tercer trimestre (33 casos) tuvieron mal resultado perinatal: incidencia de parto prematuro $50 \%$, RCF en el gemelo sobreviviente $22 \%$ y mortalidad perinatal $13 \%$. El $78 \%$ de los gemelos sobrevivientes tuvo un desarrollo normal (29).

Otra serie, mostró un resultado satisfactorio en $92 \%$ de gemelos sobrevivientes complicados con la muerte de un feto en el primer trimestre, comparado con $60 \%$ de resultados satisfactorios cuando la muerte ocurrió en el segundo y tercer trimestre $(p<0,001)$, sin encontrar cambios significativos entre los dos últimos trimestres si se compara los casos de muerte en el segundo vs el tercer trimestre (36).

El mejor pronóstico observado en el gemelo sobreviviente cuando la muerte del hermano ocurre en el primer trimestre, se fundamenta en un proceso de hialinización, fibrosis y degeneración de las vellosidades placentarias. Este proceso bloquea las anastomosis entre el feto muerto y el feto vivo, protegiendo al gemelo sobreviviente de los elementos tromboembólicos y/o la exanguinación fetal (35).

2. Corionicidad: En una serie de 16 embarazos gemelares complicados con la muerte de un feto, se analizó los resultados por corionicidad y mostró un desarrollo normal en 38\% de gemelos sobrevivientes de embarazos monocoriales comparado con $75 \%$ en aquellos sobrevivientes de embarazos bicoriales(5).

Otra serie de 92 embarazos complicados con la muerte unifetal, mostró una mortalidad perinatal para el segundo gemelo de $58 \%$ en embarazos monocoriales, comparado con $21 \%$ en embarazos bicoriales $(p<0,01)$. En la misma serie de casos, se encontró anemia fetal en $51 \%$ de sobrevivientes de embarazos monocoriales y ningún caso en los embarazos bicoriales. La discordancia de peso igualmente fue mayor en el grupo de sobrevivientes de embarazos monocoriales (78\%) versus el grupo de sobrevivientes de embarazos bicoriales $(31 \%)(p<0,01)(22)$.

Una revisión sistemática que sintetiza algunos de los datos mencionados y añade información de otros estudios, mostró mayor riesgo de muerte del feto sobreviviente si el embarazo es monocorial (OR 6,04 [IC 95\% 1,84-19,84]). La misma revisión mostró mayor riesgo de daño neurológico en sobrevivientes de embarazos monocoriales comparado con sobrevivientes de embarazos bicoriales (OR 4,07 [IC 95\% 1,32-12,51]) (37).

\section{CONSECUENCIAS EN LA EMBARAZADA}

La asociación entre feto muerto retenido in útero y la CID materna, se reportó por primera vez en 1950 asociado a embarazos únicos (9). En los embarazos gemelares, tiene una evolución crónica y no fulminante, explicado por transferencia de tromboplastinas desde el feto muerto a la madre, que activa la vía extrínseca de la cascada de coagulación, llevando al consumo de plaquetas y factores de la coagulación $(9,38)$.

En embarazos gemelares, se reportó desde 0 hasta un $14 \%$ de alteraciones en la analítica de la coagulación $(14,19,39,40)$, siendo la hipofibrinogenemia la más frecuente $(10,24)$; la hipofibrinogenemia alcanza su nadir a las cuatro a cinco semanas de la muerte fetal, con recuperación total dentro de 48 horas posparto $(9,41)$.

\section{PREVENCIÓN}

El embarazo múltiple es un embarazo de alta complejidad, incluye el elevado riesgo de mortalidad fetal in útero, que puede presentarse incluso a pesar de un control prenatal estricto. La etiología de la muerte de un gemelo, como se explicó previamente, es variable y afecta principalmente a los embarazos gemelares monocoriales.

Las anastomosis vasculares presentes en la placenta de los embarazos monocoriales, causan en algunos casos, cambios significativos del volumen sanguíneo en cada gemelo. Este desequilibrio hemodinámico causa complicaciones como STFF, perfusión arterial reversa (TRAP) y transfusión fetal aguda asociado a la muerte intrauterina de uno de los fetos.

EI STFF afecta al $20 \%$ de los embarazos monocoriales (42) y causa una mortalidad de los dos fe- 
tos de hasta $70 \%$ (43). Se ha desarrollado un tratamiento efectivo con técnica láser para disminuir las anastomosis vasculares, aumentando la sobrevida de un gemelo en $30 \%$ y de ambos en $50-60 \%$ (43). Además, es una terapia protectora de la transfusión aguda del gemelo sobreviviente, cuando el embarazo se complica con la muerte unifetal $(44,45)$.

La RCF selectiva se presenta hasta en el $25 \%$ de los embarazos gemelares, tanto monocoriales como bicoriales (42). La prevención de la muerte de ambos gemelos depende de la edad de inicio del síndrome y de la posibilidad del equipo de neonatología para recibir fetos prematuros. Cuando la muerte de un gemelo afectado por RCF se produce en un embarazo monocorial, hay riesgo de secuelas en el gemelo sobreviviente por la presencia de anastomosis vasculares placentarias. En estos casos, se plantea recibir dos fetos prematuros, un gemelo enfermo y uno sano, o esperar la muerte del gemelo enfermo para ganar EG en el gemelo sano, exponiéndole al potencial riesgo de la teoría tromboembólica y de hipoperfusión, sin embargo, este manejo es sugerible para ganar edad gestacional en los embarazos bicoriales cuando los fetos son extremadamente prematuros.

Un estudio que mostró un aumento significativo de muerte de un gemelo sin causa en el tercer trimestre $(4,6 \%)$, sugiere que la interrupción del embarazo más temprana en los embarazos monocoriales biamnióticos, entre 32 a 34 semanas, es una forma de prevención que equilibra el riesgo de muerte intrauterina y las complicaciones derivadas de la prematurez (21).

\section{MANEJO}

El manejo del embarazo gemelar complicado por la muerte de un gemelo depende principalmente de la edad gestacional cuando ocurre la muerte y de la corionicidad del embarazo.

Intervención en el primer trimestre. Dado el buen pronóstico sobre el gemelo sobreviviente, cuando la muerte de su compañero ocurre en el primer trimestre, es recomendable continuar el control del embarazo como embarazo único. Los recién nacidos de estos embarazos, si bien estadísticamente presentan un peso al nacer más bajo cuando se comparan con embarazos únicos $(p<0,001)$, no tienen mayor frecuencia de RCF ni aumento en la morbi/mortalidad $(46,47)$.

Intervención en el segundo trimestre en embarazo gemelar monocorial. El manejo del gemelo sobreviviente parte de la base de dos riesgos, la prematurez y la alteración neurológica secundaria a anemia fetal por transfusión aguda hacia el gemelo muerto o al paso de elementos tromboembólicos desde el feto muerto.

Existe una revisión de 12 casos de embarazos gemelares monocoriales con diagnóstico de STFF, donde uno de los gemelos falleció dentro de 3 días de manejo con láser (6 casos), amniodrenaje (4 casos), ambos (1 caso) o láser con coagulación del cordón (1 caso) con edad gestacional desde 17 a 26 semanas, en los que se realizó una cordocentesis para evaluar anemia fetal dentro de las primeras 24 horas. En 6 casos se necesitó una transfusión (grupo con terapia láser $1 / 6$, amniodrenaje $3 / 4$ y procedimientos combinados $2 / 2$ ), los resultados se siguieron con ecografía más Doppler semanal y RM a las 32 semanas de EG, al nacimiento con examen físico completo y ecografía transfontanelar, y el último control al año. De los 12 infantes, 10 tuvieron desarrollo adecuado hasta el año de seguimiento, uno tuvo leucomalacia periventricular a las 34 semanas donde se decidió su interrupción y otro al mes de nacimiento (27).

Se piensa que la terapia láser bloquea las anastomosis vasculares placentarias, por lo tanto protege al gemelo sobreviviente de la hipoperfusión por exanguinación, y evita el paso de material tromboembólico desde el feto muerto hacia el feto vivo (aunque esta teoría prácticamente se ha dejado de lado).

Si bien se ha propuesto como razonable hacer una cordocentesis al feto sobreviviente, para evaluar la existencia de anemia aguda y efectuar una transfusión intrauterina, el no conocer con exactitud el momento en que se produce el daño, parece muy agresivo someter a todos los fetos sobrevivientes a una cordocentesis, pues el daño al momento del procedimiento ya podría estar establecido.

Un estudio evaluó la utilidad de medir la velocidad del peak sistólico de la arteria cerebral media (VPS - ACM), para el diagnóstico de anemia fetal luego de la muerte de un gemelo en 20 embarazos monocoriales, comparado con la cordocentesis. Se confirmó 10 casos de anemia fetal por cordocentesis (gold estándar), mostrando una sensibilidad y especificidad de $90 \%$, con una tasa de falsos negativos de $10 \%$ para la evaluación ecográfica de la VPS - ACM (45), con estos datos se calculó el likelihood ratio (LR) positivo 9 y LR negativo 0,11 . En resumen, la medición de la VPS - ACM es una técnica efectiva para diagnosticar anemia fetal y disminuye el número de intervenciones invasivas innecesarias.

Intervención en el segundo trimestre en embarazo gemelar bicorial. El feto sobreviviente de un embarazo gemelar bicorial complicado con la muerte de un gemelo, está protegido del daño fetal secunda- 
rio a elementos tromboembólicos y a hipoperfusión por anemia aguda, por tener placentas individuales para cada feto. El mayor riesgo presente en los sobrevivientes de los embarazos bicoriales deriva de la prematurez (23).

\section{EVALUACIÓN NEUROLÓGICA}

Ecografía. Un estudio de 43 embarazos gemelares complicados con la muerte de un feto, no encontró diferencias significativas por corionicidad en los hallazgos ecográficos cerebrales al nacimiento, sin embargo, mostró que el 90,9\% de los recién nacidos menores de 32 semanas presentó algún tipo de alteración ecográfica cerebral, a diferencia de aquellos en edades gestacionales mayores, donde solo hubo $35 \%(p<0,001)(14)$.

Otro estudio, mostró una diferencia significativa de los hallazgos ecográficos entre embarazo monocorial y bicorial, con alteraciones en el examen reportado en fetos de embarazos monocoriales (22), sugiriendo que el daño neurológico fetal es secundario a hipoperfusión por anemia aguda.

Resonancia magnética (RM). La RM permite una mejor evaluación de las anormalidades de la corteza y materia blanca de origen isquémico (48). En una evaluación de 21 casos, 7 reportaron alteraciones por RM con una correlación de $57 \%$ con la ecografía, que solo detectó 3 casos (49).

Las lesiones isquémicas pueden aparecer como áreas focales o difusas con aumento de la señal en T2. Una evaluación de la utilidad de la RM, describe la importancia de un examen inicial y repetirlo por lo menos 2 semanas después para detectar lesiones subagudas o crónicas en el feto sobreviviente (50). Se explica que un examen normal no está exento de daño fetal, sin embargo, da mejor pronóstico no tener lesiones $(14,20)$.

\section{INTERRUPCIÓN DEL EMBARAZO}

Dada la escasa información y las series de seguimiento con muestras muy pequeñas, sugerimos crear un plan de manejo individualizado, partiendo por la evaluación de la corionicidad y EG al momento de detectar la muerte de uno de los gemelos. La decisión del momento de interrupción en estos embarazos requiere un equilibrio entre evitar el daño secundario a un medio hostil in útero y el riesgo de daño secundario a la prematurez $(51,52)$.

Un estudio mostró que el intervalo al parto fue mayor en los fetos sanos, 11,1 semanas comparado con 5,3 semanas en el grupo con secuelas $(p=0,0001)$ (36). Un reporte de un caso, con interrupción dentro de los 30 minutos de la muerte de un gemelo, no logró mejoría del resultado perinatal (53). Estos resultados, acompañados de la falta de conocimiento exacto del momento del daño multiorgánico, sugieren que el parto inmediato no sería la mejor elección para mejorar el pronóstico (39), el cual tampoco se favorece con una vigilancia estricta (14).

Luego de las 24 semanas y hasta las 34 semanas, la colocación de corticoides para madurez pulmonar y manejo conservador hasta las 36 semanas ha sido descrito independientemente de la corionicidad (39), sin embargo, pareciera ser un manejo más factible para embarazos bicoriales (23). En embarazos monocoriales, sería razonable esperar hasta las 32-34 semanas, para sobrepasar el mayor riesgo de secuelas derivadas de la prematurez, balanceando con el mayor riesgo de muerte en el tercer trimestre (21). Otros reportes avalan la interupción inmediata, si el diagnóstico de muerte fetal se da en embarazos con más de 34 semanas (14, 23). Existen indicaciones de interrupción inmediata, como las alteraciones en las evaluaciones ecográficas, pruebas de bienestar fetal y enfermedad materna concomitante (10).

En cuanto a la vía de parto, se ha visto que la cesárea no mejora los resultados perinatales, por lo tanto se debe privilegiar el parto vaginal, reservando la interrupción por cesárea a aquellas indicaciones obstétricas habituales $(10,14)$.

\section{CONTROL MATERNO}

Respecto a la madre, se sabe con seguridad que el riesgo de coagulopatía está presente solo a partir de las 4-5 semanas posteriores a la muerte fetal, siempre que el feto tenga más de 20 semanas. Por lo tanto, una analítica basal con tiempo de protrombina, tiempo de tromboplastina parcial activada, fibrinógeno y recuento plaquetario, es lo indicado. Luego el estudio seguirá cada semana, especialmente en presencia de fibrinógeno menor a $250 \mathrm{mg} / \mathrm{dL}(10,14,54)$. Si bien se reportó un caso, donde la terapia con heparina ayudó a revertir la CID y prolongar el embarazo hasta alcanzar madurez pulmonar, sin mayor riesgo materno (55), esta es una terapia que debe ser discutida en cada caso en particular. No se recomienda la evaluación del dímero $\mathrm{D}$ o productos de degradación del fibrinógeno por estar elevados basalmente durante el embarazo (54).

Adicionalmente resaltamos la necesidad de brindar un soporte emocional a los padres y aportar la información necesaria que ayude a tener un mejor control del embarazo $(14,23)$. En la Figura 2, indicamos un algoritmo de manejo para enfrentar 


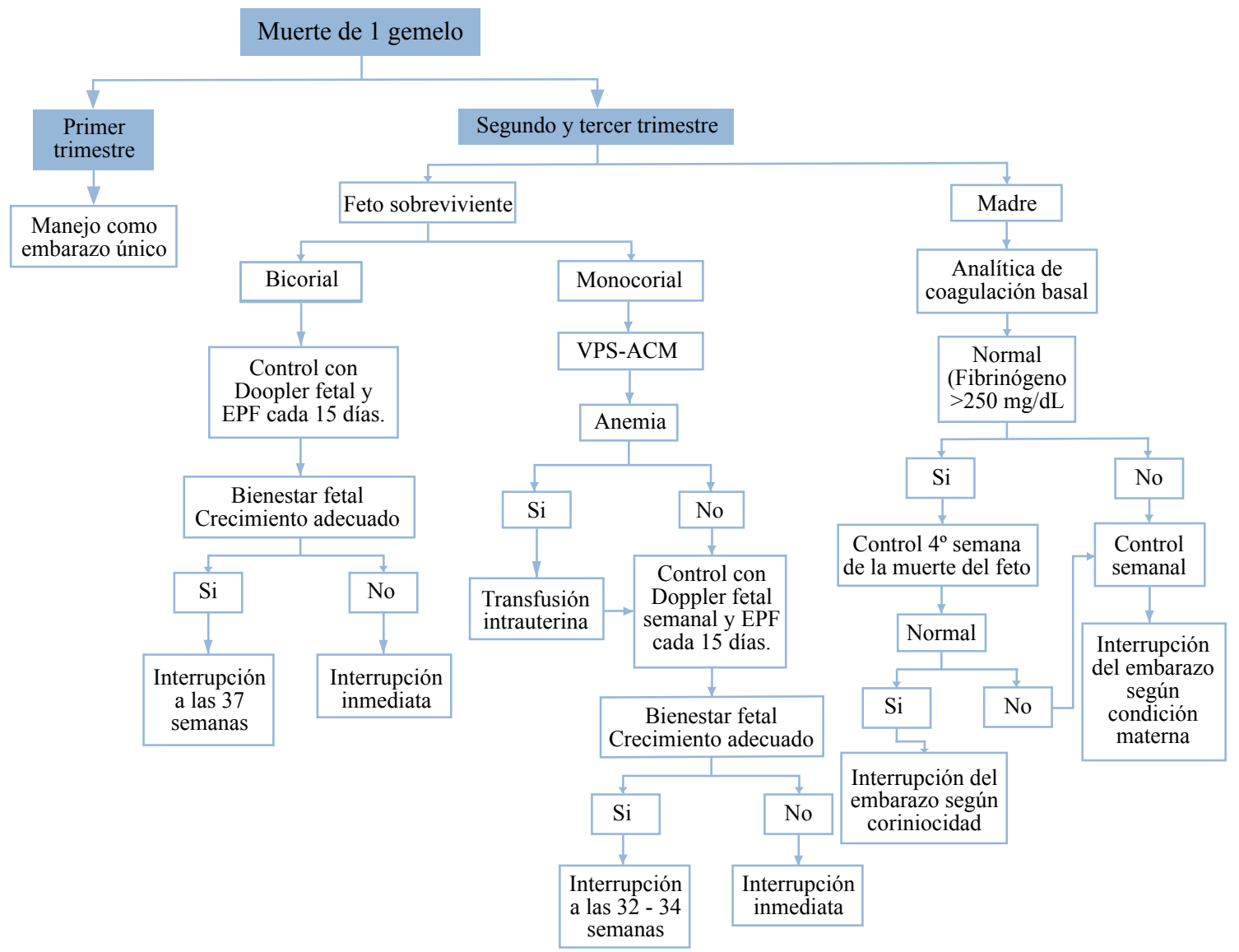

Figura 2. Algoritmo de manejo del embarazo gemelar complicado con la muerte de un feto in útero.

esta situación clínica.

\section{CONCLUSIONES}

El manejo del embarazo gemelar complicado con la muerte de un feto in útero es desafiante, entre otras razones pues la mejor evidencia disponible es resultado de reportes de casos y series pequeñas. El diagnóstico temprano de la corionicidad, la edad gestacional, y la patología fetal y obstétrica, permiten optimizar el manejo de los embarazos complicados con la muerte unifetal para mejorar los resultados del gemelo sobreviviente y la madre.

\section{REFERENCIAS}

1. Blickstein I, Goldman RD, Mazkereth R. Incidence and birth weight characteristics of twins born to mothers aged 40 years or more compared with 35-39 years old mothers: a population study. J Perinat Med
2001;29:128-32.

2. ICMART. World Collaborative Report on Assisted Reproductive Technology, 2002. Hum Reprod 2009;24:2310-20.

3. Nazer J, Aguila A, Cifuentes L. La frecuencia de nacimientos de gemelos aumentó en un hospital chileno coincidiendo con el consumo periconcepcional de harina fortificada con ácido fólico. Rev Méd Chile 2006;134:48-52.

4. Murphy KW. Intrauterine death in a twin: implications for the survivor. In: Ward RH, Whittle M, editors. Multiple pregnancy. London: RCOG Press;1995:218-30.

5. Fusi L, Gordon H. Twin pregnancy complicated by single intrauterine death. Problems and outcome with conservative management. $\mathrm{Br} \mathrm{J}$ Obstet Gynaecol 1990;97:511-6.

6. Lee YM, Wylie BJ, Simpson LL, D'Alton ME. Twin chorionicity and the risk of stillbirth. Obstet Gynecol 2008;111(2 Pt 1):301-8.

7. Sampson A. Vanishing twins: the frequency of spontaneous fetal reduction of a twin pregnancy. Ultrasound 
Obstet Gynecol 1992;2:107-9.

8. Cherouny P, Hoskins I, Johnson T, Niebyl J. Multiple pregnancy with late death of one fetus. Obstet Gynecol 1989;74:318-20.

9. Woo HHN, Sin SY, Tang LCH. Single foetal death in twin pregnancies: review of the maternal and neonatal outcomes and management. HKMJ 2000;6:293-300.

10. Villalobos N, López C. Síndrome del gemelo muerto. Rev Obstet Ginecol Venez 2002;62:11-5.

11. Posner AC, Klein MA. Fetus papyraceus. Obstet Gynecol 1954;3:106-10.

12. Dictionary Mosby's Medical. 8th edition. 2009. Hallado en: http://medical-dictionary.thefreedictionary.com/ fetal+maceration.

13. Saito K, Ohtsu Y, Amano K, Nishijima M. Perinatal outcome and management of single fetal death in twin pregnancy: A case series and review. J Perinat Med 1999;27:473-7.

14. Cruceyra M, de La Calle M, Rodriguez R, Magdaleno Dans F, González A. Pronóstico materno y perinatal en la gestación gemelar con muerte de un gemelo intraútero: estudio retrospectivo. Toko-Gin Pract 2011;70:58-62.

15. Axt R, Mink D, Hendrik J, Ertan K, von Blohn M, Schmidt W. Maternal and neonatal outcome of twin pregnancies complicated by single fetal death. J Perinat Med 1999;27:221-7.

16. Pharoah POD. Neurological outcome in twins. Semin Neonatol 2002;7:223-30.

17. Landy HJ, Keith LG. The vanishing twin: a review. Human Reprod Update 1998;4:177-83.

18. Kilby MD, Govind A, O'Brien PM. Outcome of twin pregnancies complicated by a single intrauterine death: a comparison with viable twin pregnancies. Obstet Gynecol 1994;84:107-9.

19. Petersen IR, Nyholm HC. Multiple pregnancies with single intrauterine demise. Description of twentyeight pregnancies. Acta Obstet Gynecol Scand 1999;78:202-6.

20. Simonazzi G, Segata M, Ghi T, Sandre F, Ancora G, Bernardi B, Tani G, Rizzo N, Santini D, Bonasoni P, Pilu G. Accurate neurosonographic prediction of brain injury in the surviving fetus after the death of a monochorionic cotwin. Ultrasound Obstet Gynecol 2006;27:517-21.

21. Barigye O, Pasquini L, Galea P, Chambers H, Chappell L, Fisk N. High risk of unexpected late fetal death in monochorionic twins despite intensive ultrasound surveillance: a cohort study. PLoS Medicine 2005;2(e172):521-7.

22. Bajoria R, Wee LY, Anwar S, Ward S. Outcome of twin pregnancies complicated by single intrauterine death in relation to vascular anatomy of the monochorionic placenta. Hum Reprod 1999;14:2124-30.

23. Hillman SC, Morris RK, Kilby MD. Single twin demise: consequence for survivors. Sem Fetal Neonat Med 2010;15:319-26.

24. Moore CM, McAdams AJ, Sutherland J. Intrauterine disseminated intravascularcoagulation: A syndrome of multiple pregnancy with a dead twin fetus. J Pediatr 1969;74:523-8.

25. Fusi L, Mc Parland P, Fisk P, Nicolini U, Wiggleswor- th J. Acute twin-twin transfusion syndrome: posible mechanism for brain damaged survivors after intrauterine death of a monochorionic twin. Obstet Gynecol 1991;78 517-20.

26. Okamura K, Murotsuki J, Tanigawara S, Uehara S, Yajima A. Funipuncture for evaluation of hematologic and coagulation indices in the surviving twin following co-twin's death. Obstet Gynecol 1994;83:975-8.

27. Senat MV, Bernard JP, Loizeau S, Ville Y. Management of single fetal death in twin-to-twin transfusion syndrome: a role for fetal blood sampling. Ultrasound Obstet Gynecol 2002;20:360-3.

28. Nicolini U, Pisoni MP, Cela E, Roberts A. Fetal blood sampling immediately before and within 24 hours of death in monochorionic twin pregnancies complicated by single intrauterine death. Am J Obstet Gynecol 1998;179:800-3.

29. Prömpeler H, Madjar H, Klosa W, Du Bois A, Zahradnik $\mathrm{H}$, Schillinger $\mathrm{H}$, Breckwoldt $M$. Twin pregnancies with single fetal death. Acta Obstet Gynecol Scand 1994;73:205-8.

30. van Heteren CF, Nijhuis JG, Semmekrot BA, Mulders $L G$, van den Berg PP. Risk for surviving twin after fetal death of co-twin in twin-twin transfusion syndrome. Obstet Gynecol 1998;92:215-9.

31. Gaucherand P, Rudigoz RC, Piacenza JM. Monofetal death in multiple pregnancies: risks for the co-twin, risk factors and obstetrical management. Eur J Obstet Gynecol Reprod Biol 1994;55:111-5.

32. Glinianaia SV, Pharoah POD, Wright C, Rankin JM. Fetal or infant death in twin pregnancy: neurodevelopmental consequence for the survivor. Arch Dis Child Fetal Neonatal Ed 2002;86:F9-15.

33. Pharoach POD, Adi Y. Consecuences of in-utero death in a twin pregnancy. The Lancet 2000;355:1597602.

34. Williams K, Hennessy E, Alberman E.Cerebral palsy: effects of twinning, birthweigt and gestational age. Arch Dis Child 1996;75:F178-82.

35. Mohazath HR, Spigos DG, Anciaux D, Nadimapalli V. Fetus papyraceus. AJR 1993;160:665-6.

36. Nicolini U, Poblete A. Single intrauterine death in monochorionic twin pregnancies. Ultrasound Obstet Gynecol 1999;14:297-301.

37. Ong SSC, Zamora J, Khan KS, Kilby MD. Prognosis for the co-twin following single-twin death: a systematic review. BJOG 2006;113:992-8.

38. Landy $\mathrm{HJ}$, Weingold AB. Management of a multiple gestation complicated by an antepartum fetal demise. Obstet Gynecol Surv 1989;44:171-6.

39. Fichera A, Zambolo C, Accorsi P, Martelli P, Ambrosi $C$, Frusca T. Perinatal outcome and neurological follow up of the cotwins in twin pregnancies complicated by single intrauterine death. Eur J Obstet Gynecol Reprod Biol 2009;147:37-40.

40. Kaufman HK, Hume RF Jr, Calhoun BC, Carlson N, Yorke V, Elliott D, Evans MI. Natural history of twin gestation complicated by in utero fetal demise: associations of chorionicity, prematurity, and maternal morbidity. Fetal Diagn Ther 2003;18:442-6.

40. Cunningham FG, Leveno KJ, Bloom SL, Hauth JC, Rouse DJ, Spong CY. Chapter 35. Obstetrical He- 
morrhage. Williams Obstetrics, 23e. http://www.accessmedicine.com/content.aspx?alD=60.

41. Denbow ML, Cox P, Taylor M, Hammal DM, Fisk NM. Placental angioarchitecture in monochorionic twin pregnancies: Relationship to fetal growth, fetofetal transfusion syndrome and pregnancy outcome. Am J Obstet Gynecol 2000;182:417-26.

42. Moise K, Johnson A. Management of twin-twin transfusion syndrome. UpToDate 19.1.2011. Hallado en: http://www.uptodate.com/contents/management-oftwin-twin-transfusion-syndrome?source=see_link .

43. O'Donoghue K, Rutherford M, Engineer N, Wimalasundera R, Cowan F, Fisk N. Transfusional fetal complications after single intrauterine death in monochorionic multiple pregnancy. BJOG 2009;116:804-12.

44. Senat MV, Loizeau S, Couderc S, Bernard JP, Ville $Y$. The value of middle cerebral artery peak systolic velocity in the diagnosis of fetal anemia after intrauterine death of one monochorionic twin. Am J Obstet Gynecol 2003; 189:1320-4.

45. Pinborg A, Lidegaard O, la Cour Freiesleben N, Andersen AN. Consequences of vanishing twins in IVF/ ICSI pregnancies. Human Reprod 2005;20:2821-9.

46. Shebl O, Ebner T, Sommergruber M, Sir A, Tews G. Birth weight is lower for survivors of the vanishing twin syndrome: a case-control study. Fertil Steril 2008;90:310-4.

47. Garel C, Delezoide AL, Elmaleh-Berges M, Menez F, Fallet-Bianco C, Vuillard E, Luton D, Oury JF, Sebag G. Contribution of fetal MR imaging in the evalua- tion of cerebral ischemic lesions. Am $\mathrm{J}$ Neuroradiol 2004;25:1563-8.

48. Jelin AC, Norton ME, Bartha AI, Fick A, Glenn O. Intracranial magnetic resonance imaging findings in the surviving fetus after spontaneous monochorionic cotwin demise. Am J Obstet Gynecol 2008;199:398. e1-398-5.

49. Glenn O. MR imaging of the fetal brain. Pediatr Radiol 2010;40:68-81.

50. Evans MI, Lau TK. Making decisions when no good choices exist: delivery of the survivor after intrauterine death of the co-twin in monochorionic twin pregnancies. Fetal Diagn Ther 2010;28:191-5.

51. Hanna JH, Hill JM. Single intrauterine fetal demise in multiple gestation. Obstet Gynecol 1984;63:126-30.

52. Karageyim Karsidag AY, Kars B, Dansuk R, Api O, Unal O, Turan MC, Goynumer G. Brain damage to the survivor within 30 min of co-twin demise in monochorionic twins. Fetal Diagn Ther 2005;20:91-5.

53. Poblete A, Carvajal J, Ferreira F, Kushner L, Donoso B, Durán R, Robles C, Oyarzún E. Embarazo gemelar con un óbito: Rescate mediante transfusión intravascular intrauterina. Rev Chil Obstet Ginecol 2004;69:239-41.

54. Romero R, Duffy TP, Berkowitz RL, Chang E, Hobbins JC. Prolongation of a preterm pregnancy complicated by death of a single twin in utero and disseminated intravascular coagulation. Effects of treatment with heparin. N Engl J Med 1984;310:772-4. 